\title{
ZF OFDM Receiver for Underwater Communications
}

\author{
Alain Y. Kibangou, Cyrille Siclet, and Laurent Ros
}

\begin{abstract}
In this paper, we propose a new scheme of transmission and reception of OFDM ( Orthogonal Frequency Division Multiplexing) signals for underwater wireless communications. The transmitter makes use of a kind of superimposed pilot signals, which are used by the receiver for estimating both the channel parameters (paths gain and delay) and the Doppler. Then, the estimated parameters are used for estimating the informative symbols in the zero forcing (ZF) sense. A new estimation scheme based on the resolution of two harmonic retrieval problems is proposed. Unlike standard methods, data resampling and estimation of residual carrier frequency offset are avoided. The efficiency of the proposed scheme is evaluated by means of simulations.
\end{abstract}

Index Terms-Underwater acoustic communications, OFDM, Doppler distorsion, Hankel matrix, Vandermonde decomposition, Total least squares.

\section{INTRODUCTION}

Nowadays, a great interest is dedicated to study fleets of autonomous underwater vehicles (AUV) working together to reach a common objective such as a gradient search and following for source detection. For this purpose derivation of distributed, multi-vehicle co-ordination schemes and development of efficient underwater acoustic communication protocols are needed. For achieving high data rate and large system capacity, Orthogonal Frequency Division Multiplexing (OFDM) has been claimed to be an efficient communication technology. It allows designing low complexity receivers to deal with highly dispersive channels. This facts motivates the use of OFDM in underwater environments.

Underwater acoustic channels are wideband in nature due to the small ratio of carrier frequency to the signal bandwidth, which introduces frequency-dependent Doppler shifts [1]. They also exhibit several propagation paths.

In order to adequately recover the transmitted information, algorithms at the receiver must include estimation and compensation of the Doppler scaling factor, channel estimation, and information symbols estimation. Several approaches have been suggested in the literature for estimating the Doppler scaling factor. They are based on the use of preamble and postamble of a packet consisting of multiple OFDM blocks

Alain Y. Kibangou is with GIPSA-Lab, System control Department, University Joseph Fourier, CNRS, 961 rue de la Houille Blanche, B.P. 46- 38402 Saint Martin d'Hères, France (e-mail: Alain.Kibangou@gipsa-lab.grenobleinp.fr)

Cyrille Siclet and Laurent Ros are with GIPSA-Lab, Image and Signal Department, University Joseph Fourier, CNRS, 961 rue de la Houille Blanche, B.P. 46- 38402 Saint Martin d'Hères, France (e-mail: Cyrille.Sicletu @ gipsalab.grenoble-inp.fr, Laurent.Ros@ gipsa-lab.grenoble-inp.fr)

This work was supported by the ANR and the EU funded projects CONNECT and FeedNetBack
[1] or by exploiting correlation induced by the cyclic prefix [2]. Then, the received signal is resampled by using a sampling period related to the estimated Doppler scaling factor. It is also necessary to estimate and compensate for the residual carrier frequency offset (CFO) since the Doppler can vary between consecutive OFDM blocks inside a given packet.

In this paper, the received data are processed block-byblock. We make use of high resolution harmonic estimation methods to estimate both Doppler scaling factor and channel parameters (path gains and delays). The advantage of the proposed scheme is to avoid data resampling and residual CFO estimation and compensation. The estimated channel parameters and Doppler are then used to build a channel matrix that is used for estimating the informative symbols following a zero forcing scheme.

Notation: We denote by $\mathscr{V}(\mathbf{x}, N)$ the $L \times N$ Vandermonde matrix with $\mathbf{x} \in \mathbb{C}^{L}$ as generator. Its first column is constituted by $1 \mathrm{~s}$.

\section{SignAL MODEL}

The proposed receiver is based on block-by-block processing. We assume that consecutive OFDM symbols are separated by a sufficient guard interval in order to avoid intersymbol interference. Moreover, the transmitter and the receiver clocks are synchronized.

In the transmission scheme considered in this paper, the signal $s($.$) , results on the superposition of two OFDM sig-$ nals $s_{0}($.$) and s_{1}($.$) , i.e. s(t)=s_{0}(t)+s_{1}(t)$. The information contained in $s_{0}($.$) is known to the receiver and will serve$ to estimate the channel parameter, whereas $s_{1}($.$) contains the$ informative symbols unknown to the receiver. The transmitted bandpass signal is then given by:

$$
s(t)=\sum_{i=0}^{1} s_{i}(t), \text { with } s_{i}(t)=\operatorname{Re}\left\{\sum_{k \in \mathscr{K} i} c_{k, i} e^{j 2 \pi f_{k, i} t} \Pi_{T_{i}}\left(t-\bar{\tau}_{i}\right)\right\}
$$

where:

- $\mathscr{K}_{i}=\left\{-K_{i} / 2, \cdots, K_{i} / 2-1\right\}, K_{i}$ being the number of subcarriers used for transmitting the learning sequence $\left\{c_{k, 0}\right\}$, if $i=0$, or the informative sequence $\left\{c_{k, 1}\right\}$, if $i=1$.

- $f_{k, i}=f_{c}+k \Delta f_{i}$, are the subcarrier frequencies, $\Delta f_{i}$ being the frequency spacing between two consecutive subcarriers and $f_{c}$ is the (central) carrier frequency.

- $\Pi_{T_{i}}(t)=1$ if $t \in\left[0, T_{i}\right]$ and 0 otherwise.

- $\bar{\tau}_{0}=0$ whereas $\bar{\tau}_{1}$, which will be defined later, stands for the delaying operation of the informative signal $s_{1}($.$) with$ respect to the learning signal $s_{0}($.$) .$ 
The signal bandwidth equals $B=K_{1} \Delta f_{1}=K_{1} / T_{1}$. We assume that less subcarriers are used for transmitting the pilot sequence than the informative one, i.e. $K_{0}<K_{1}$. Moreover, we have $\Delta f_{0}=B / K_{0}$, meaning that the frequency spacing in $s_{0}$ is more important than in $s_{1}($.$) .$

The signal $s($.$) is transmitted to the receiver through an$ underwater acoustic channel modeled as:

$$
h(t, \tau)=\sum_{p=1}^{P} A_{p}(t) \delta\left(t-\tau_{p}(t)\right)
$$

where $A_{p}(t)$ and $\tau_{p}(t)$ are respectively the real-valued gain and the delay associated with the $p$ th propagation path. In addition, the following assumptions are adopted:

- All paths are affected by a similar Doppler scaling factor $a$ such that

$$
\tau_{p}(t)=\tau_{p}-a t
$$

- The path delays $\tau_{p}$, the gains $A_{p}$, and the Doppler scaling factor $a$ are constant over the block duration $T=T_{0}+T_{1}+$ $T_{g}-\bar{\tau}_{1}$.

- The maximal path delay $\tau_{p}$ is known and the guard interval $T_{g}$ is sufficiently large to avoid interference between consecutive blocks. We have

$$
T_{g}>\max \left\{\tau_{p}\right\}=\tau_{\max }
$$

The analytic representation of the received signal $y(t)$ is then given by:

$$
\begin{aligned}
y(t)= & \sum_{i=0}^{1} \sum_{k_{i} \in \mathscr{K}_{i}} \sum_{p=1}^{P} c_{k_{i}, i} A_{p} \Pi_{T_{i}}\left((1+a) t-\tau_{p}-\bar{\tau}_{i}\right) \\
& \times e^{-j 2 \pi f_{k_{i}, i} \tau_{p}} e^{j 2 \pi f_{k_{i}, i}(1+a) t} .
\end{aligned}
$$

Assuming that the bounds of the path delays, $\tau_{\min }{ }^{1}$ and $\tau_{\max }$, and that of the Doppler scaling factor are known ${ }^{2}$. We get:

$$
\tau_{\min } \leq \tau_{p} \leq \tau_{\max }, \quad \lambda_{\min } \leq 1+a \leq \lambda_{\max } .
$$

Therefore, for $t \in\left(\frac{T_{0}+\tau_{\max }}{\lambda_{\min }}, T\right)$ and $\bar{\tau}_{1} \geq \lambda_{\min } T-\tau_{\max }-T_{1}$, we get the signal part conveying the informative data:

$$
y_{1}(t)=\sum_{k_{1} \in \mathscr{K}_{1}} h_{k_{1}}(t) c_{k_{1}, 1}
$$

with

$h_{k_{1}}(t)=\sum_{p=1}^{P} A_{p} \Pi_{T_{1}}\left((1+a) t-\tau_{p}-\bar{\tau}_{1}\right) e^{-j 2 \pi f_{k_{1}, 1} \tau_{p}} e^{j 2 \pi f_{k_{1}, 1}(1+a) t}$

By sampling the received signal at the period $T_{e} \geq \frac{1}{B}$, equation

(2) can be written in matrix form as follows:

$$
\mathbf{y}=\mathbf{H c}
$$

where $\mathbf{y}, \mathbf{H}$, and $\mathbf{c}$ contain respectively the samples of received signal corresponding to $t \in\left(\frac{T_{0}+\tau_{\max }}{\lambda_{\min }}, T\right)$, the channel matrix, whose entries are defined in (3), and the informative symbols.

\footnotetext{
${ }^{1}$ The knowledge of the minimal path delay is difficult. Therefore, we get the absolute minimum $\tau_{\min }=0$.

${ }^{2} \lambda_{\max }$ and $\lambda_{\min }$ are related to the maximal and minimal velocities of the underwater vehicles, which can be a priori known.
}

Therefore, if the channel parameters and the Doppler factor are known, the $\mathrm{ZF}$ receiver is obtained by solving

$$
\hat{c}=\left(\mathbf{H}^{H} \mathbf{H}\right)^{-1} \mathbf{H}^{H} \mathbf{y} .
$$

In the following section, we derive a joint channel and Doppler estimation method.

\section{DOPPLER AND CHANNEL ESTIMATION}

Let us select $t_{0}$ and $t_{1}$ such that

$$
\frac{\tau_{\text {max }}}{\lambda_{\text {min }}} \leq t_{0}<t_{1} \leq \frac{T_{0}+\tau_{\text {min }}}{\lambda_{\text {max }}} .
$$

For $t \in\left[t_{0}, t_{1}\right]$, the received signal can be written as:

$$
y(t)=\sum_{k_{0} \in \mathscr{K}_{0}} c_{k_{0}, 0} B_{k_{0}} e^{j 2 \pi f_{k_{0}, 0}(1+a) t}
$$

with

$$
B_{k_{0}}=\sum_{p=1}^{P} A_{p} e^{-j 2 \pi f_{k_{0}, 0} \tau_{p}}
$$

In discrete-time, we get:

$$
y_{n}=\sum_{k_{0} \in \mathscr{K}_{0}} c_{k_{0}, 0} B_{k_{0}} e^{j 2 \pi f_{k_{0}, 0}(1+a) n T_{e}} .
$$

Inside the observation window defined by the bounds (6), the received signal can be viewed as a mixture of $K_{0}$ complex valued harmonics with constant magnitudes. As a consequence, the magnitudes $c_{k_{0}, 0} B_{k_{0}}$ and the phases $2 \pi f_{k_{0}, 0}(1+a) T_{e}$ can be obtained by solving a harmonic retrieval problem. From (8), one can note that the magnitude $B_{k_{0}}$ can also be viewed as a mixture of $P$ harmonics. So, for estimating both the Doppler scaling factor, the channel gains and delays, we have to solve two harmonic retrieval problems. In the literature, the harmonic retrieval problem have been extensively studied [3][8]. In this paper, we derive new estimators by making use of HTLS (Hankel Total Least Squares) [8], [9], a high resolution method. In the sequel, we first give the main guidelines for solving the harmonic retrieval problem before deriving our estimators.

\section{A. Harmonic retrieval using HTLS [8]}

Let us consider the samples $z_{q}$ such that $z_{q}=\sum_{k=1}^{K} \alpha_{k} e^{j \varphi_{k} q}, q=$ $q_{0}, \cdots, q_{0}+N-1$. By setting $N=L+M-1, L>Q, M \geq Q$, we first build the Hankel matrix $\mathbf{Z} \in \mathbb{C}^{L \times M}$ having respectively as first column and last row the vectors

$$
\mathbf{Z}_{.1}=\left(\begin{array}{llll}
z_{q_{0}} & z_{q_{0}+1} & \cdots & z_{q_{0}+L-1}
\end{array}\right)^{T}
$$

and

$$
\mathbf{Z}_{L .}=\left(\begin{array}{llll}
z_{q_{0}+L-1} & z_{q_{0}+L} & \cdots & z_{q_{0}+N-1}
\end{array}\right) .
$$

It admits the two following decompositions (10) and (11):

$$
\mathbf{Z}=\mathbf{S} \operatorname{diag}(\boldsymbol{\alpha}) \mathbf{T}^{T}
$$

with $\boldsymbol{\alpha}=\left(\alpha_{1}, \cdots, \alpha_{K}\right)^{T}, \mathbf{S}^{T}=\mathscr{V}(\boldsymbol{\phi}, L), \mathbf{T}^{T}=\mathscr{V}(\boldsymbol{\phi}, M), \boldsymbol{\phi}=$ $\left(e^{j \varphi_{1}}, \cdots, e^{j \varphi_{K}}\right)^{T}$, and

$$
\mathbf{Z}=\mathbf{U} \mathbf{\Sigma} \mathbf{V}^{H}
$$


where $\mathbf{U} \in \mathbb{C}^{L \times K}, \mathbf{V} \in \mathbb{C}^{M \times K}$, and $\boldsymbol{\Sigma} \in \mathbb{C}^{K \times K}$ contain respectively the left and right singular vectors and the singular values of $\mathbf{Z}$. From (10) and (11), we deduce that $\mathbf{U}$ and $\mathbf{S}$ generate the same subspace. Hence, it exists a nonsingular matrix $\mathbf{Q} \in \mathbb{C}^{K \times K}$ such that $\mathbf{U}=\mathbf{S Q}$. The Vandermonde matrix $\mathbf{S}$ possesses the shift-invariance property expressed as: $\underline{\mathbf{S}} \operatorname{diag}(\boldsymbol{\phi})=\overline{\mathbf{S}}$, where the line on the bottom (resp. on the top) of a matrix stands for deleting the first (resp. the last) row. We also have:

$$
\underline{\mathbf{U}}=\underline{\mathbf{S}} \mathbf{Q}, \quad \overline{\mathbf{U}}=\overline{\mathbf{S}} \mathbf{Q}
$$

By combining these two above equations, we get:

$$
\overline{\mathbf{U}}=\underline{\mathbf{U}} \mathbf{Q}^{-1} \operatorname{diag}(\boldsymbol{\phi}) \mathbf{Q} .
$$

Hence, the poles $e^{j \varphi_{k}}$ are the eigenvalues of $\boldsymbol{\Phi}=$ $\mathbf{Q}^{-1} \operatorname{diag}(\boldsymbol{\phi}) \mathbf{Q}$. Therefore, we have to estimate $\boldsymbol{\Phi}$ and then deducing its eigenvalues. $\boldsymbol{\Phi}$ is estimated by solving $\overline{\mathbf{U}}=\underline{\mathbf{U}} \boldsymbol{\Phi}$ in the total least squares sense. We get:

$$
\hat{\boldsymbol{\Phi}}=-\mathbf{W}_{12} \mathbf{W}_{22}^{-1}
$$

where

$$
\mathbf{W}=\left(\begin{array}{ll}
\mathbf{W}_{11} & \mathbf{W}_{12} \\
\mathbf{W}_{21} & \mathbf{W}_{22}
\end{array}\right) \in \mathbb{C}^{2 K \times 2 K}
$$

is the matrix containing the right singular vectors of ( $\left.\begin{array}{ll}\underline{\mathbf{U}} & \overline{\mathbf{U}}\end{array}\right)$.

Once the poles estimated, the magnitude $\alpha_{k}$ can be obtained as the least squares solution of the vectorized Vandermonde decomposition (10):

$$
\operatorname{vec}(\mathbf{Z})=(\mathbf{T} \odot \mathbf{S}) \boldsymbol{\alpha},
$$

where $\odot$ denotes the Khatri-Rao product, the poles in $\mathbf{S}$ and $\mathbf{T}$ being replaced by their estimated values.

\section{B. Doppler scaling factor estimation}

Let us consider the $N$ samples $y_{n}, n=n_{0}, \cdots, N+n_{0}-1$, of the received signal $y_{n}$ as given in (9). Herein, the pole and the magnitude of the involved harmonics are $\phi_{k_{0}}=e^{j 2 \pi f_{k_{0}, 0}(1+a) T_{e}}$ and $\alpha_{k_{0}}=c_{k_{0}, 0} B_{k_{0}} e^{j 2 \pi f_{k_{0}, 0}(1+a) n_{0} T_{e}}$.

Let us denote by $\hat{\phi}_{k_{0}}$ the estimated poles using the HTLS algorithm. If the sampling period $T_{e}$ is chosen such that

$$
T_{e} \leq \frac{1}{2 \lambda_{\max } \max \left\{f_{k_{0}, 0}\right\}}
$$

then the angle of $\hat{\phi}_{k_{0}}$, denoted $\angle \hat{\phi}_{k_{0}}$, belongs to $[-\pi, \pi]$. In fact, the estimated eigenvalues equal the actual poles up to a permutation. For removing such an ambiguity, the estimated eigenvalues can be sorted with their pulsation in an ascending order. Therefore, we deduce the following estimator for the Doppler scaling factor:

$$
\hat{a}=-1+\frac{1}{K_{0}} \sum_{k_{0} \in \mathscr{K}_{0}} \frac{\angle \hat{\phi}_{k_{0}}}{2 \pi f_{k_{0}, 0} T_{e}}
$$

\section{Path delays and gains estimation}

Let us denote $\hat{\alpha}_{k_{0}}$ the magnitudes obtained by solving (14), where the poles in $\mathbf{S}$ and $\mathbf{T}$ have been replaced by their estimated values. We get:

$$
\hat{B}_{k_{0}}=\frac{c_{k_{0}, 0}^{*}}{\left|c_{k_{0}, 0}\right|^{2}} \hat{\alpha}_{k_{0}} e^{-j 2 \pi f_{k_{0}, 0}(1+\hat{a}) n_{0} T_{e}}, \quad k_{0} \in \mathscr{K}_{0} .
$$

Note that we can rewrite $B_{k_{0}}$, defined in (8), as

$$
B_{k_{0}}=\sum_{p=1}^{P} A_{p} e^{-j 2 \pi f_{-K_{0} / 2,0} \tau_{p}} e^{-j 2 \pi k_{0} \Delta f_{0} \tau_{p}}, \quad k_{0} \in \mathscr{K}_{0} .
$$

Provided, $K_{0} \geq 2 P$, we can solve this harmonic retrieval problem using the HTLS method, the poles and the magnitudes being $e^{-j 2 \pi \Delta f_{0} \tau_{p}}$ and $A_{p} e^{-j 2 \pi f_{-K_{0} / 2,0} \tau_{p}}$ respectively.

Let us denote $\hat{\beta}_{p}$ the estimated harmonic magnitudes using HTLS. We can then estimate the gains $A_{p}$ as the magnitudes

$$
\hat{A}_{p}=\left|\hat{\beta}_{p}\right| \text {. }
$$

We also deduce $\theta_{p}=\frac{\hat{\beta}_{p}}{\left|\hat{\beta}_{p}\right|}=e^{-j 2 \pi f_{-K_{0} / 2,0} \tau_{p}}$.

The estimation of the path delays is a more complicate task. Indeed, we cannot guarantee that $2 \pi \Delta f_{0} \tau_{p}$ belongs to $[-\pi, \pi]$. As a consequence, we cannot directly deduce the value of the path delays from the angle of the entries $\hat{\psi}_{p}$ of $\hat{\boldsymbol{\beta}}$. However, it exists an integer $m_{0}$ such that

$$
\angle \hat{\psi}_{p}=-2 \pi \Delta f_{0} \tau_{p}+2 \pi m_{0} .
$$

For different integers $m$, we define a set of possible path delays $\tau_{p, m}$ such that $\tau_{p, m}<T_{g}$ :

$$
\tau_{p, m}=-\frac{\angle \hat{\psi}_{p}}{2 \pi \Delta f_{0}}+\frac{m}{\Delta f_{0}} \quad \text { and } \quad \theta_{p, m}=e^{-j 2 \pi f_{-K_{0} / 2} \tau_{p, m}} .
$$

Let us now consider the square distance

$$
\kappa_{p, m}=\left|\theta_{p}-\theta_{p, m}\right|^{2}=\left|1-e^{-j 2 \pi f_{-K_{0} / 2} \frac{m^{\prime}-m}{\Delta f_{0}}}\right|^{2}
$$

After few manipulations, it can be shown that this square distance is minimal only for $\tau_{p, m}=\tau_{p}$, the actual path delay:

$$
\hat{\tau}_{p}=\arg \min \kappa_{p, m} .
$$

\section{Simulation RESUlts}

In these simulations, the range of frequency used by the underwater vehicles was $[15 \mathrm{kHz}-28 \mathrm{kHz}]$. We considered Doppler scaling factor lower than $10^{-3}$, meaning that the maximal relative speed was $1.5 \mathrm{~m} / \mathrm{s}$. The carrier frequency was set equal to $f_{c}=21 \mathrm{kHz}$, whereas the guard interval was $T_{g}=10 \mathrm{~ms}$. We used $K_{0}=16$ sub-carriers for the learning sequence and $K_{1}=512$ for the informative one. The duration of the learning sequence was $T_{0}=2 T_{g}$ whereas that of the informative sequence was $T_{1}=46.54 \mathrm{~ms}$. The sampling frequency was $1 / T_{e}=56.056 \mathrm{kHz}$. We made use of the QPSK modulation. All the results presented below are averaged values over 100 independent Monte Carlo runs. The additive noise was a complex valued white Gaussian noise. The paths gain and delay of the simulated channel are depicted in figure 1. 


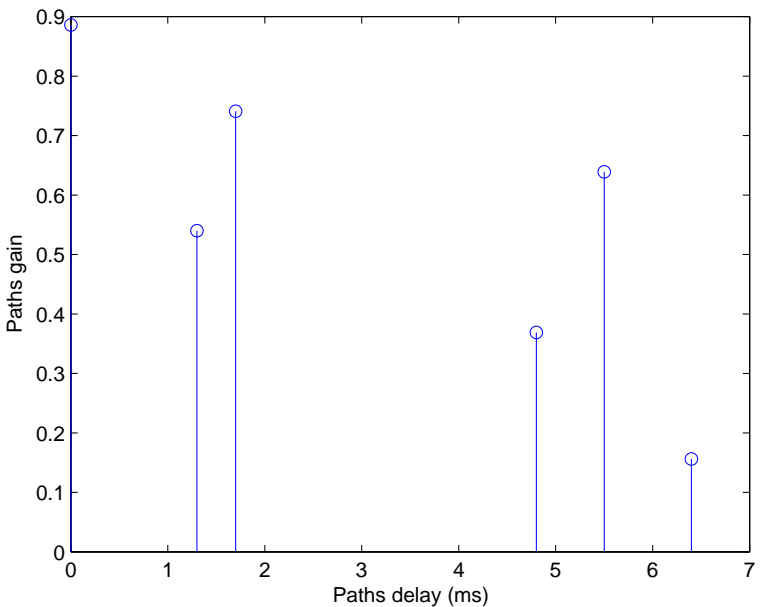

Figure 1. Paths gain and delay of the simulated channel.

We evaluate the performance of the derived ZF receiver in terms of BER (Bit Error Rate). Figure 2 and 3 depict the mean of the BER for channels with $P=4$ and $P=6$ for two different values of the Doppler scaling factor. We compare the performance of the equalization method when the Doppler and the channel parameters are estimated by the method proposed herein and with the ideal case, i.e. the actual parameters are used for constructing the channel matrix. In the ideal case, by increasing the SNR, we get better performance for a channel with $P=6$ than that with $P=4$. Since, the first channel has more propagation paths, the received signal is more redundant and therefore allows improving the informative sequence estimation. When using the estimated parameters, we don't have the same behaviour. Indeed, with the same amount of data, the parameter estimation is degraded by increasing the number of propagation paths. Moreover, the gap in performance between the ideal case and the estimated one increases with the number of propagation paths.

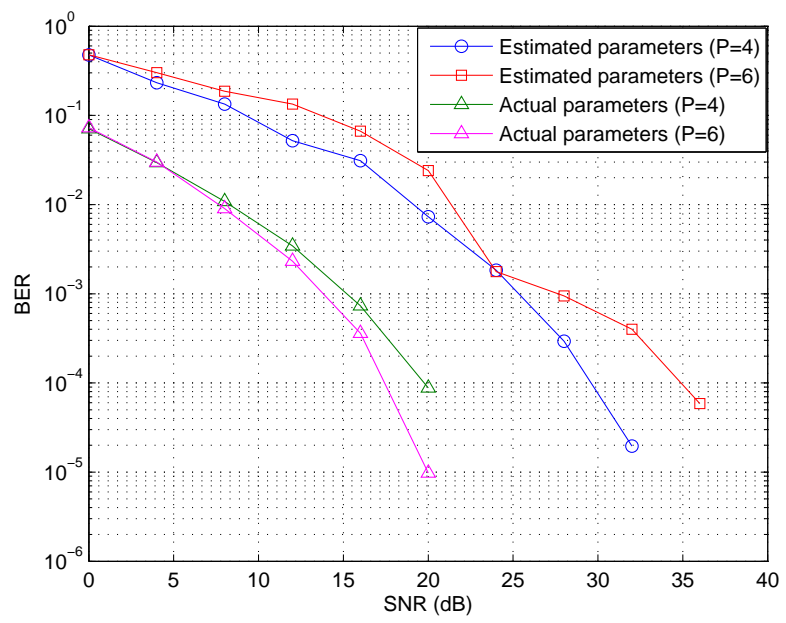

Figure 2. BER obtained with the proposed estimation method $(a=6.67 \times$ $\left.10^{-4}\right)$.

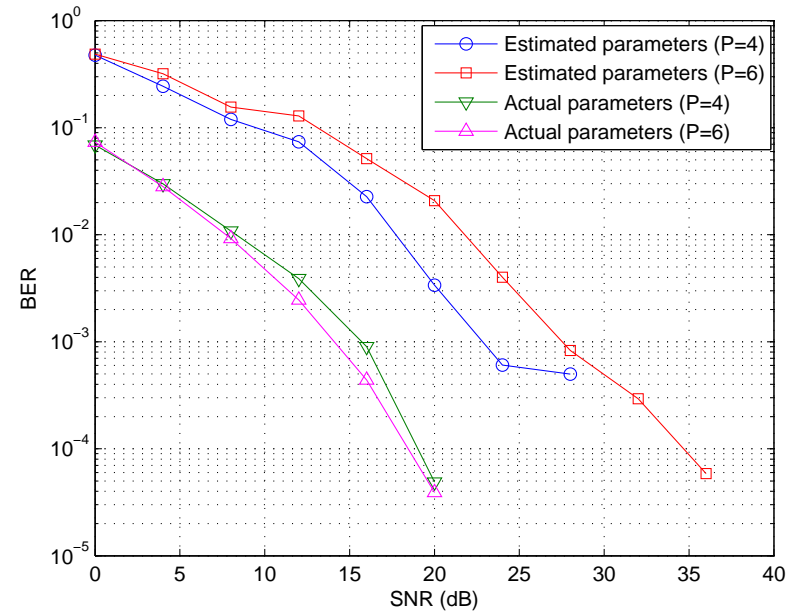

Figure 3. BER obtained with the proposed estimation method $(a=3.33 \times$ $\left.10^{-4}\right)$.

\section{CONCLUSION}

In this paper, we have presented a new zero-forcing OFDM receiver for underwater acoustic communications. In the proposed scheme, each OFDM symbol is constituted by a the superposition of two OFDM sub-blocks characterized by different number of subcarriers. The first OFDM sub-block is used for estimating the channel parameters and the Doppler scaling factor. The estimation methods are based on high resolution methods for solving harmonic retrieval problems. Then, the estimated parameters are used for retrieving the informative symbols according to the Zero forcing criterion. The proposed scheme is particularly efficient for moderate to high SNR. Robustness to noise, for lower levels of SNR, should be investigated. Extension to multiusers underwater communications is under investigation.

\section{REFERENCES}

[1] B. Li, S. Zhou, M. Stojanovic, L. Freitag, and P. Willett, "Multicarrier communication over underwater acoustic channels with nonuniform doppler shifts," IEEE Journal of Oceanic Engineering, vol. 33, no. 2, pp. 198-209, 2008.

[2] B.-C. Kim and I-T. Lu, "Parameter study of OFDM underwater communications system," in Proc. of OCEANS 2000 MTS/IEEE Conference and Exhibition, September 11-14 2000, vol. 2, pp. 1251-1255.

[3] S.M. Kay, Modern spectral estimation, Theory and Application, PrenticeHall, Englewood Cliffs, New Jersey, USA, 1988.

[4] R. Roy and T. Kailath, "ESPRIT-Estimation of signal parameters via rotational invariance techniques," IEEE Trans. on Signal Proc., vol. 37, no. 7, pp. 984-995, July 1989.

[5] G.T. Zhou and G.B. Giannakis, "On estimating random amplitudemodulated harmonics using higher order spectra," IEEE Journal of Oceanic Engineering, vol. 19, no. 4, pp. 529-539, October 1994.

[6] B. Friedlander and J.M Francos, "Estimation of amplitude and phase parameters of multicomponent signals," IEEE Trans. on Signal Proc., vol. 43, no. 4, pp. 917-926, April 1995.

[7] P. Stoica and R. Moses, Introduction to spectral analysis, Prentice-Hall, Englewood Cliffs, New Jersey, USA, 1997.

[8] J.M. Papy, L. De Lathauwer, and S. Van Huffel, "Exponential data fitting using multilinear algebra: The single-channel and multi-channel case," Numer. Linear Algebra Appl., vol. 12, pp. 809-826, 2005.

[9] S. Van Huffel, "Enhanced resolution based on minimum variance estimation and exponential data modeling," Signal Processing, vol. 33, no. 3, pp. 333-355, 1993. 